“C 2019 IEEE. Personal use of this material is permitted. Permission from IEEE must be obtained for all other uses, in any current or future media, including reprinting/republishing this material for advertising or promotional purposes, creating new collective works, for resale or redistribution to servers or lists, or reuse of any copyrighted component of this work in other works." 


\title{
Generating Quantum States of Surface Plasmon-Polariton Pairs with a Nonlinear Nanoparticle
}

\author{
Nikita Olekhno ${ }^{1}$, Mihail Petrov ${ }^{1}$, Ivan Iorsh $^{1}$, Andrey A. Sukhorukov ${ }^{2}$, Alexander Solntsev $^{3}$ \\ 1. Research Center of Nanophotonics and Metamaterials, ITMO University, Saint Petersburg 199034, Russia \\ 2. Nonlinear Physics Centre, Research School of Physics and Engineering, The Australian National University, Canberra ACT 2601, Australia \\ 3. School of Mathematical and Physical Sciences, University of Technology Sydney, Sydney NSW 2007, Australia
}

In the last few years, materials with strong second-order optical nonlinearity such as gallium arsenide, barium titanate and transition metal dichalcogenides have attracted significant attention, because they for the first time allowed efficient nonlinear optical interactions on the sub-micron scales. One of such nonlinear optical interactions - spontaneous parametric down-conversion (SPDC) - allows the generation of pairs of correlated photons and can enable photon entanglement [1]. This is the foundation of many quantum optical applications ranging from secure communication to ultrafast quantum computing [2]. The key challenges in this field are efficiency and the generation of on-demand quantum states.

(a)

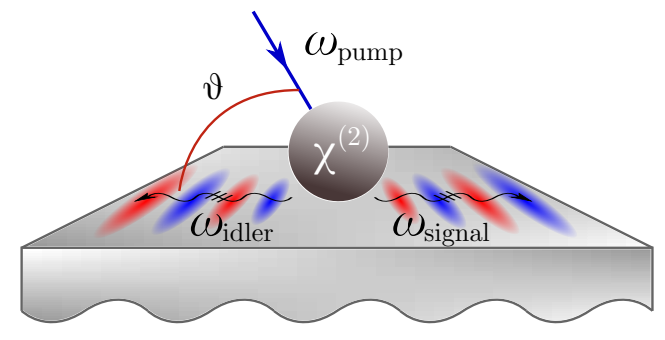

(b)

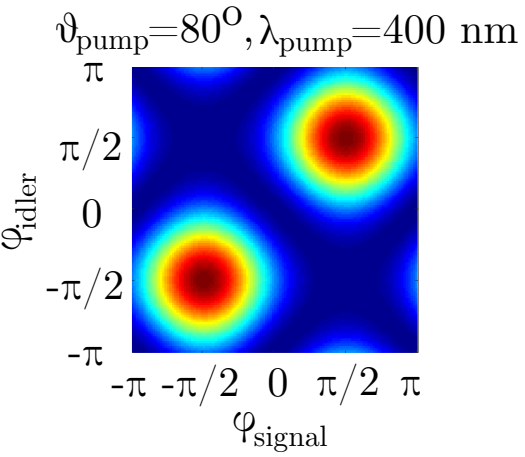

Fig. 1 (a) Generation of SPP pairs by a GaAs [001] nanoparticle near the silver-air interface. (b) Angular correlations between the generated signal and idler SPPs with $\omega_{i, s}=\omega_{\text {pump }} / 2$ demonstrating the so-called $N 00 N$-state in the angular variables $\varphi_{i, s}$ measured from the positive $x$-direction. The distance between the detection points and the nanoparticle is $12 \mu \mathrm{m}$. The radius of the nanoparticle is $10 \mathrm{~nm}$, the pump is linearly polarized in the TM geometry within the $(x, z)$ plane and the pump wave vector is $\theta$ with respect to the interface.

In this work, we report the theoretical study on the generation of surface plasmon-polariton (SPP) pairs in a $\chi^{(2)}$ nanoparticle placed in the vicinity of the metal-dielectric interface, Fig. 1(a), following the general formalism of Ref.[3]. Here, the SPDC enhancement is attributed to the resonant nature of SPPs instead of eigenmodes of the nanoparticle, which may increase overall efficiency while substituting photons by plasmons. We demonstrate that one can obtain N00N-states of SPP pairs by using a GaAs nanoparticle, Fig. 1(b). The best picture of the N00N-state is achieved at the normal incidence of the pump, and the pump angle $\theta=80^{\circ}$ is shown to demonstrate the robustness of the N00N-state generation. This approach also allows us to tailor two-plasmon quantum states by exploiting the well-manifested spin momentum locking of light, which results in the unidirectional excitation of surface plasmons. We perform the classification of quantum states of SPP pairs in the far-field region and compare several materials including gallium arsenide, barium titanate and transition metal dichalcogenides for their potential use as sources of nonlinearity for controllable generation of various two-plasmon quantum states.

\section{References}

[1] G. Marino et al, "Sum-Frequency- and Photon-Pair-Generation in AlGaAs Nano-Disks", OSA Technical Digest (online), paper NpM21.2 (2018).

[2] A.S. Solntsev and A.A. Sukhorukov, "Path-entangled photon sources on nonlinear chips", Reviews in Physics 2, 19-31 (2017).

[3] A.N. Poddubny, I.V. Iorsh, A.A. Sukhorukov, "Generation of Photon-Plasmon Quantum States in Nonlinear Hyperbolic Metamaterials", Phys. Rev. Lett. 117, 123901 (2016). 